\title{
A COMPREHENSIVE REVIEW ON AJEYA GHRITA AN AGADA PREPARATION
}

\author{
Prajwal H N ${ }^{1}$, Nataraj $\mathrm{H} \mathrm{R}^{2}$, Deepa $\mathrm{P}^{3}$, Anushree C G ${ }^{4}$, Gazala Hussain ${ }^{5}$ \\ ${ }^{1} P G$ Scholar, \\ ${ }^{2}$ Associate Professor, \\ ${ }^{3,4}$ PG Scholar, Dept. of Agada Tantra, \\ ${ }^{5}$ Associate Professor, Dept. of Rasashastra \& Bhaishajya Kalpana, \\ Sri Dharmasthala Manjunatheshwara College of Ayurveda \& Hospital, Hassan
}

Corresponding Author:_Prajwal H N,PG Scholar, Dept. of Agada Tantra,Sri Dharmasthala Manjunatheshwara College of Ayurveda \& Hospital, Hassan

Article DOI: $\underline{\text { https://doi.org/10.36713/epra6774 }}$

DOI No: 10.36713/epra6774

\begin{abstract}
Agada tantra is one of the unique branches among the eight branches of Ayurveda which deals with different poisoning conditions, diagnosis, and treatment. In Ayurveda Garavisha( artificial poison) is mentioned as a type of Visha( poison) which is formed by the combination of two or more poisonous or non-poisonous substances, which slowly gets accumulated in the body and ultimately affects all parts of the body by vitiating all Dosha( humor), Dhatu( tissues), Mala( $\sim$ waste products) and Srotas( channels). Many ayurvedic formulations are mentioned for the treatment of Garavisha. Ajeya ghrita is one among them which is explained in Ashtanga Sangraha. It is a form of Ghrita Kalpa ( ghee preparation), the use of Ghrita ( poison) plays an important role in the management of Visha ( poison) condition. Ajeya ghrita is a formulation that has a combination of many herbs, in which most of the herbs possess Vishahara ( antipoison) property. The ingredients are easily accessible and have a simple method of preparation may encourage the physician to use this formulation in the management of Visha ( poison) condition. Hence an effort is made to review the Ajeya ghrita for Vishahara ( anti-poison) property.
\end{abstract}

KEYWORDS: Ajeya ghrita, Ayurveda, Garavisha, Ghrita, Visha.

\section{INTRODUCTION}

Agada tantra is a unique branch among the eight branches of Ayurveda. It mainly deals with Visha ( poison) with its types, signs and symptoms, diagnosis, and management with various formulations. 'Gada' means Roga ( disease) and 'Agada' means Roga Pratikara ( to fight against disease) ${ }^{1}$. In Ayurveda, the Garavisha ( artificial poison) is considered as a type of Kritrima Visha ( artificial poison). Acharya Vagbhata opines that Garavisha ( $\sim$ artificial poison) is the poison which is formed from the combination of different parts of animals and excreta or combination of medicines or Bhasmas ( ash preparations) which are having opposite properties or the poisons having the less potency ${ }^{2}$.

In Ayurveda for Garavisha ( artificial poison) treatment various Agada formulations are described by various Acharyas. Ajeya ghrita is one among them which is described by Acharya Vagbhata. It is described in Ashtanga Sangraha in the context of Garavisha Chikitsa ( treatment of artificial poison). Ajeya Agada is a Ghrita Kalpa ( ghee preparation) that contains 25 herbs in it. 


\section{EPRA International Journal of Research and Development (IJRD)}

Volume: 6 | Issue: 7 | July 2021

- Peer Reviewed Journal

Table 1: INGREDIENTS OF AJEYA GHRITA

\begin{tabular}{|c|c|c|c|c|c|}
\hline $\begin{array}{c}\text { Sl. } \\
\text { No. }\end{array}$ & Dravya (Drug) & Rasa (Taste) & $\begin{array}{c}\text { Guna } \\
\text { (Properties) }\end{array}$ & $\begin{array}{c}\text { Veerya } \\
\text { (Potency) }\end{array}$ & $\begin{array}{c}\text { Vipaka } \\
\text { (Metabolic } \\
\text { property) }\end{array}$ \\
\hline 1 & $\begin{array}{l}\text { Madhuka }{ }^{3} \\
\text { (Madhuca longifolia) }\end{array}$ & $\begin{array}{l}\text { Madhura (sweet), } \\
\text { Kashaya (astringent) }\end{array}$ & $\begin{array}{l}\text { Guru (heavy), } \\
\text { Snigdha } \\
\text { (unctuous) }\end{array}$ & Sheeta (cold) & $\begin{array}{l}\text { Madhura } \\
\text { (sweet) }\end{array}$ \\
\hline 2 & $\begin{array}{l}\text { Tagara }{ }^{4} \\
\text { (Valeriana wallichii) }\end{array}$ & $\begin{array}{l}\text { Tikta (bitter) } \\
\text { Katu (pungent) } \\
\text { Kashaya } \\
\text { (astringent) }\end{array}$ & $\begin{array}{l}\text { Laghu (light) } \\
\text { Snigdha } \\
\text { (Unctuous) }\end{array}$ & $\begin{array}{l}\text { Ushna } \\
\text { (hot) }\end{array}$ & $\begin{array}{l}\text { Katu } \\
\text { (pungent) }\end{array}$ \\
\hline 3 & $\begin{array}{l}\text { Kushta } \\
\text { (Saussurea lappa) }\end{array}$ & $\begin{array}{l}\text { Tikta (bitter) } \\
\text { Katu (pungent) } \\
\text { Madhura (sweet) }\end{array}$ & $\begin{array}{l}\text { Laghu (light) } \\
\text { Ruksha (dry) } \\
\text { Tikshna (sharp) }\end{array}$ & $\begin{array}{l}\text { Ushna } \\
\text { (hot) }\end{array}$ & $\begin{array}{l}\text { Katu } \\
\text { (pungent) }\end{array}$ \\
\hline 4 & $\begin{array}{l}\text { Bhadradaru } \\
\text { (Cedrus deodara) }\end{array}$ & $\begin{array}{l}\text { Tikta (bitter) } \\
\text { Katu (pungent) } \\
\text { Kashaya } \\
\text { (astringent) }\end{array}$ & $\begin{array}{l}\text { Laghu (light) } \\
\text { Ruksha (dry) }\end{array}$ & $\begin{array}{l}\text { Ushna } \\
\text { (hot) }\end{array}$ & $\begin{array}{l}\text { Katu } \\
\text { (pungent) }\end{array}$ \\
\hline 5 & $\begin{array}{l}\text { Harenu } \\
\text { (Pisum sativum) }\end{array}$ & $\begin{array}{l}\text { Madhura (sweet) } \\
\text { Tikta (bitter) } \\
\text { Kashaya } \\
\text { (astringent) }\end{array}$ & Ruksha (dry) & Sheeta (cold) & $\begin{array}{l}\text { Madhura } \\
\text { (sweet) }\end{array}$ \\
\hline 6 & $\begin{array}{l}\text { Manjishta }{ }^{8} \\
\text { (Rubia cordifolia) }\end{array}$ & $\begin{array}{l}\text { Madhura (sweet) } \\
\text { Tikta (bitter) }\end{array}$ & $\begin{array}{l}\text { Guru (heavy), } \\
\text { Ruksha (rough) }\end{array}$ & Ushna (hot) & Katu (pungent) \\
\hline 7 & $\begin{array}{l}\text { Ela } \\
\text { (Elattariacardamomum) }\end{array}$ & $\begin{array}{l}\text { Katu (pungent) } \\
\text { Madhura(sweet) }\end{array}$ & $\begin{array}{l}\text { Laghu (light) } \\
\text { Ruksha (dry) }\end{array}$ & $\begin{array}{l}\text { Sheeta } \\
\text { (cold) }\end{array}$ & Katu (pungent) \\
\hline 8 & $\begin{array}{l}\text { Elavaluka }^{I 0} \\
\text { (Prunus cerasus) }\end{array}$ & $\begin{array}{l}\text { Kashaya } \\
\text { (astringent) }\end{array}$ & Laghu (light) & $\begin{array}{l}\text { Sheeta } \\
\text { (cold) }\end{array}$ & Katu (pungent) \\
\hline 9 & $\begin{array}{l}\text { Nagapushpa }{ }^{11} \\
\text { (Mesua ferra) }\end{array}$ & $\begin{array}{l}\text { Kashaya } \\
\text { (astringent) } \\
\text { Tikta (bitter) }\end{array}$ & $\begin{array}{l}\text { Ruksha (dry) } \\
\text { Tikshna (sharp) } \\
\text { Laghu (light) }\end{array}$ & $\begin{array}{l}\text { Ushna (hot)/ } \\
\text { Anushna (Not } \\
\text { hot) }\end{array}$ & Katu (pungent) \\
\hline 10 & $\begin{array}{l}\text { Utpala }^{12} \\
\text { (Nymphea alba) }\end{array}$ & $\begin{array}{l}\text { Madhura (sweet) } \\
\text { Tikta (bitter) } \\
\text { Kashaya } \\
\text { (astringent) }\end{array}$ & $\begin{array}{l}\text { Laghu (light) } \\
\text { Snigdha } \\
\text { (Unctuous) } \\
\text { Picchila } \\
\text { (sliminess) }\end{array}$ & $\begin{array}{l}\text { Sheeta } \\
\text { (cold) }\end{array}$ & $\begin{array}{l}\text { Madhura } \\
\text { (sweet) }\end{array}$ \\
\hline 11 & $\begin{array}{l}\text { Plava }^{13} \\
\text { (Cyperus scariosus) }\end{array}$ & $\begin{array}{l}\text { Tikta (bitter) } \\
\text { Katu (pungent) } \\
\text { Kashaya } \\
\text { (astringent) }\end{array}$ & $\begin{array}{l}\text { Laghu (light) } \\
\text { Ruksha (dry) }\end{array}$ & $\begin{array}{l}\text { Sheeta } \\
\text { (cold) }\end{array}$ & $\begin{array}{l}\text { Katu } \\
\text { (pungent) }\end{array}$ \\
\hline 12 & $\begin{array}{l}\text { Vidanga }^{14} \\
\text { (Embelia ribes Burm.) }\end{array}$ & $\begin{array}{l}\text { Katu (pungent) } \\
\text { Kashaya } \\
\text { (astringent) }\end{array}$ & $\begin{array}{l}\text { Laghu (light) } \\
\text { Ruksha (dry) } \\
\text { Tikshna (sharp) }\end{array}$ & Ushna (hot) & $\begin{array}{l}\text { Katu } \\
\text { (pungent) }\end{array}$ \\
\hline 13 & Chandana $^{15}$ & Tikta (bitter) & Laghu (light) & Sheeta & Katu \\
\hline
\end{tabular}

(C) 2021 EPRA IJRD | Journal DOI: https://doi.org/10.36713/epra2016 | www.eprajournals.com |42 | 
SJIF Impact Factor 2021: 8.013| ISI I.F.Value:1.241| Journal DOI: 10.36713/epra2016

ISSN: 2455-7838(Online)

EPRA International Journal of Research and Development (IJRD)

Volume: 6 | Issue: 7 | July 2021

- Peer Reviewed Journal

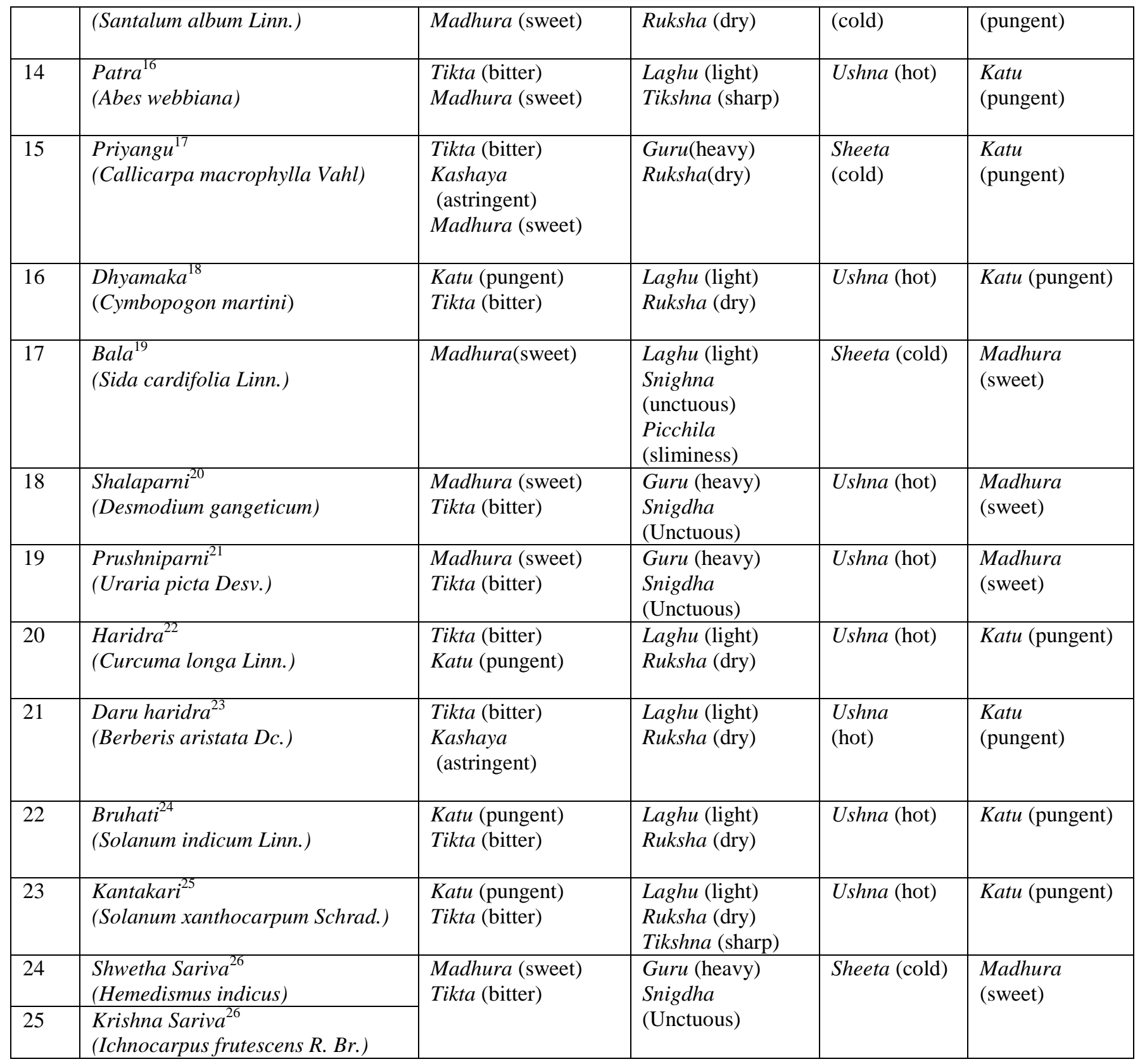

Table 2: Action \& indications

\begin{tabular}{|c|l|l|l|l|}
\hline $\begin{array}{c}\text { Sl. } \\
\text { No. }\end{array}$ & \multicolumn{1}{|c|}{ Dravya (Drug) } & $\begin{array}{l}\text { Doshaghnata (action } \\
\text { on dosha) }\end{array}$ & \multicolumn{1}{|c|}{ Karma (action) } & \multicolumn{1}{c|}{$\begin{array}{c}\text { Rogaghnata (therapeutic } \\
\text { indications) }\end{array}$} \\
\hline 1 & $\begin{array}{l}\text { Madhuka } \\
\text { (Madhuca longifolia) })\end{array}$ & $\begin{array}{l}\text { VataPittahara } \\
\text { (Alleviates Vata and } \\
\text { Pitta) }\end{array}$ & $\begin{array}{l}\text { Balya (strengthens the } \\
\text { body) } \\
\text { Shukrala (increases } \\
\text { seminal flow) }\end{array}$ & $\begin{array}{l}\text { RaktaPitta (bleeding disorder) } \\
\text { Trishna (thirst) } \\
\text { Kshaya (cachexia) }\end{array}$ \\
\hline 2 & $\begin{array}{l}\text { Tagara } \\
\text { (Valeriana wallichii) }\end{array}$ & $\begin{array}{l}\text { KaphaVatahara } \\
\text { (Alleviates Kapha and } \\
\text { Vata) }\end{array}$ & $\begin{array}{l}\text { Vishaghna (anti } \\
\text { poisonous) }\end{array}$ & $\begin{array}{l}\text { Anidra (insomnia) } \\
\text { Apasmara (epilepsy) }\end{array}$ \\
\hline
\end{tabular}

(c) 2021 EPRA IJRD | Journal DOI: https://doi.org/10.36713/epra2016 | www.eprajournals.com |43 | 
SJIF Impact Factor 2021: 8.013| ISI I.F.Value:1.241| Journal DOI: 10.36713/epra2016

ISSN: 2455-7838(Online)

EPRA International Journal of Research and Development (IJRD)

Volume: 6 | Issue: 7 | July 2021

- Peer Reviewed Journal

\begin{tabular}{|c|c|c|c|c|}
\hline 3 & $\begin{array}{l}\text { Kushta } \\
\text { (Saussurea lappa) }\end{array}$ & $\begin{array}{l}\text { VataKaphahara } \\
\text { (alleviates Vata and } \\
\text { Kapha) }\end{array}$ & $\begin{array}{l}\text { Lekhaniya (scraping) } \\
\text { Vrishya (aphrodisiac) } \\
\text { Vishaghna } \\
\text { (anti poisonous) }\end{array}$ & $\begin{array}{l}\text { Kushta (leprosy) } \\
\text { Hikka (hiccups) } \\
\text { Kasa (cough) } \\
\text { Swasa (difficulty in breathing) } \\
\text { Hridroga (cardiac diseases) } \\
\text { Kandu (itching) } \\
\text { Visarpa (erysipelas) }\end{array}$ \\
\hline 4 & $\begin{array}{l}\text { Bhadradaru } \\
\text { (Cedrus deodara) }\end{array}$ & $\begin{array}{l}\text { KaphaVatahara } \\
\text { (Alleviates Kapha and } \\
\text { Vata) }\end{array}$ & $\begin{array}{l}\text { Deepana } \\
\text { (gastrostimulant) }\end{array}$ & $\begin{array}{l}\text { Jwara (fever) } \\
\text { Kushta(skin disease) } \\
\text { Dushta Vrana(non-healing ulcer) } \\
\text { Prameha(diabetes) } \\
\text { Kandu(pruritis) } \\
\text { Krimi(worm infestation) } \\
\end{array}$ \\
\hline 5 & $\begin{array}{l}\text { Harenu }^{7} \\
\text { (Pisum sativum) }\end{array}$ & $\begin{array}{l}\text { KaphaPittahara } \\
\text { (Alleviates Kapha and } \\
\text { Pitta) }\end{array}$ & Grahi (one which holds) & $\begin{array}{l}\text { Dahapaha (relieves burning } \\
\text { sensation) } \\
\text { Pushtiprada (improves strength) }\end{array}$ \\
\hline 6 & $\begin{array}{l}\text { Manjishta } \\
\text { (Rubia cordifolia) }\end{array}$ & $\begin{array}{l}\text { KaphaPittahara } \\
\text { (Alleviates Kapha and } \\
\text { Pitta) }\end{array}$ & $\begin{array}{l}\text { Varnya } \\
\text { Vishaghna } \quad \text { (anti } \\
\text { poisonous) }\end{array}$ & $\begin{array}{l}\text { Jwara } \text { (fever) } \\
\text { Prameha } \text { (diabetes) } \\
\text { Kushta } \text { (skin disease) } \\
\text { Visarpa (Herpes) }\end{array}$ \\
\hline 7 & $\begin{array}{l}\text { Ela } \\
\text { (Elattaria } \\
\text { cardamomum) }\end{array}$ & $\begin{array}{l}\text { KaphaVatahara } \\
\text { (Alleviates Kapha and } \\
\text { Vata) }\end{array}$ & $\begin{array}{l}\text { Hridya } \\
\text { (cardioprotective) } \\
\text { Deepana } \\
\text { (gastrostumulant) }\end{array}$ & $\begin{array}{l}\text { Hridroga (cardiac disease) } \\
\text { Swasa (difficulty in breathing) } \\
\text { Kasa (cough) } \\
\text { Mutrakrichra (dysuria) } \\
\text { Chardi (emesis) } \\
\text { Arshas (piles) }\end{array}$ \\
\hline 8 & $\begin{array}{l}\text { Elavaluka }^{10} \\
\text { (Prunus cerasus) }\end{array}$ & $\begin{array}{l}\text { KaphaVatahara } \\
\text { (Alleviates Kapha and } \\
\text { Vata) }\end{array}$ & $\begin{array}{l}\text { Shukra shodhana (semen } \\
\text { purifier) }\end{array}$ & $\begin{array}{l}\text { Kandu (pruritis) } \\
\text { Vrana (wound) } \\
\text { Kushta (skin disease) } \\
\text { Murcha (unconsciousness) } \\
\text { Hridruja (cardiac disease) }\end{array}$ \\
\hline 9 & $\begin{array}{l}\text { Nagapushpa } \\
\text { (Mesua ferra) }\end{array}$ & $\begin{array}{l}\text { KaphaPittahara } \\
\text { (Alleviates Kapha and } \\
\text { Pitta) }\end{array}$ & $\begin{array}{l}\text { Vishahara (anti } \\
\text { poisonous) } \\
\text { Kushtaghna (alleviates } \\
\text { skin disease) } \\
\text { Shothahara (alleviated } \\
\text { inflammation) }\end{array}$ & $\begin{array}{l}\text { Visha roga (poisoning conditions) } \\
\text { Kushta (skin diseases) } \\
\text { Visarpa (herpes) } \\
\text { Jwara (fever) } \\
\text { Kandu (pruritis) } \\
\text { Trishna (thirst) }\end{array}$ \\
\hline 10 & $\begin{array}{l}\text { Utpala }^{12} \\
\text { (Nymphea alba) }\end{array}$ & $\begin{array}{l}\text { Tridoshahara } \\
\text { (alleviates Vata, Pitta, } \\
\text { Kapha) }\end{array}$ & $\begin{array}{l}\text { Medhya (improves } \\
\text { intellect) } \\
\text { Mutra virajana (restoring } \\
\text { normal urine color) } \\
\text { Grahi (one which holds) }\end{array}$ & $\begin{array}{l}\text { RaktaPitta (bleeding disorder) } \\
\text { Trishna (thirst) } \\
\text { Daha (burning sensation) } \\
\text { Prameha (diabetes) } \\
\text { Jwara (Fever) } \\
\text { Atisara (diarrhea) }\end{array}$ \\
\hline 11 & $\begin{array}{l}\text { Plava }^{13} \\
\text { (Cyperus scariosus) }^{\text {Cyperis }}\end{array}$ & $\begin{array}{l}\text { KaphaPittahara } \\
\text { (Alleviates Kapha and } \\
\text { Pitta) }\end{array}$ & $\begin{array}{l}\text { Deepana (gastro } \\
\text { stimulant) } \\
\text { Pachana } \\
\text { Grahi (one which holds) } \\
\text { Lekhana (scraping) }\end{array}$ & $\begin{array}{l}\text { Jwara (fever) } \\
\text { Kushta (skin disease) } \\
\text { Apasmara (epilepsy) } \\
\text { Grahani (ulcerative colitis) } \\
\text { Nidranasha (insomnia) } \\
\text { Rakta vikara (disorders of blood) }\end{array}$ \\
\hline 12 & Vidanga $^{14}$ & KaphaVatahara & Vishaghna (anti & Kushta (skin disease) \\
\hline
\end{tabular}

(C) 2021 EPRA IJRD | Journal DOI: https://doi.org/10.36713/epra2016 | www.eprajournals.com |44 | 
SJIF Impact Factor 2021: 8.013| ISI I.F.Value:1.241| Journal DOI: 10.36713/epra2016

ISSN: 2455-7838(Online)

EPRA International Journal of Research and Development (IJRD)

Volume: 6 | Issue: 7 | July 2021

- Peer Reviewed Journal

\begin{tabular}{|c|c|c|c|c|}
\hline & (Embelia ribes Burm.) & $\begin{array}{l}\text { (Alleviates Kapha and } \\
\text { Vata) }\end{array}$ & $\begin{array}{l}\text { poisonous) } \\
\text { Krimighna } \\
\text { (anthelminthic) } \\
\text { Deepana (gastro } \\
\text { stimulant) }\end{array}$ & $\begin{array}{l}\text { Udara (ascites) } \\
\text { Adhmana (bloating) } \\
\text { Krimi (worm infestation) } \\
\text { Shoola (pain) }\end{array}$ \\
\hline 13 & $\begin{array}{l}\text { Chandana }^{15} \\
\text { (Santalum album } \\
\text { Linn.) }\end{array}$ & $\begin{array}{l}\text { KaphaPittahara } \\
\text { (Alleviates Kapha and } \\
\text { Pitta) }\end{array}$ & $\begin{array}{l}\text { Varnya (skin whitening) } \\
\text { Dahaprashamana } \\
\text { (alleviates burning } \\
\text { sensation) }\end{array}$ & $\begin{array}{l}\text { Jwara (fever) } \\
\text { Kushta (skin disease) } \\
\text { RaktaPitta (bleeding disorder) } \\
\text { Trishna (thirst) } \\
\text { Daha (burning sensation) } \\
\text { Visarpa (herpes) }\end{array}$ \\
\hline 14 & $\begin{array}{l}\text { Patra }^{16} \\
\text { (Abes webbiana) }\end{array}$ & $\begin{array}{l}\text { KaphaVatahara } \\
\text { (Alleviates Kapha and } \\
\text { Vata) }\end{array}$ & $\begin{array}{l}\text { Hridya } \\
\text { (cardioprotective) } \\
\text { Deepana (gastro } \\
\text { stimulant) }\end{array}$ & $\begin{array}{l}\text { Aruchi (anorexia) } \\
\text { RaktaPitta (bleeding disorder) } \\
\text { Kshaya (cachexia) } \\
\text { Shwasa (dyspnea) }\end{array}$ \\
\hline 15 & $\begin{array}{l}\text { Priyangu } \\
\text { (Callicarpa } \\
\text { macrophylla Vahl) }\end{array}$ & $\begin{array}{l}\text { KaphaPittahara } \\
\text { (Alleviates Kapha and } \\
\text { Pitta) }\end{array}$ & $\begin{array}{l}\text { Mutra virajana (restoring } \\
\text { normal urine color) } \\
\text { Purisha sangrahaniya } \\
\text { (bowel binding) }\end{array}$ & $\begin{array}{l}\text { RaktaPitta (bleeding disorder) } \\
\text { Trishna (thirst) } \\
\text { Daha (burning sensation) } \\
\text { Jwara (fever) } \\
\text { Prameha (diabetes) }\end{array}$ \\
\hline 16 & $\begin{array}{l}\text { Dhyamaka }^{18} \\
\text { (Cymbopogon martini) }^{\text {Cymbogon }}\end{array}$ & $\begin{array}{l}\text { KaphaVatahara } \\
\text { (Alleviates Kapha and } \\
\text { Vata) }\end{array}$ & $\begin{array}{l}\text { Sthanya janana } \\
\text { ( Galactogogues) }\end{array}$ & $\begin{array}{l}\text { Jwara (fever) } \\
\text { Daha (burning sensation) } \\
\text { Trishna (excessive thirst) } \\
\text { Chardi (emesis) } \\
\text { Kasa (cough) } \\
\text { Swasa (difficulty in breathing) } \\
\text { Krimi (microbial disorder) } \\
\text { Arshas (piles) }\end{array}$ \\
\hline 17 & $\begin{array}{l}\text { Bala }^{19} \\
\text { (Sida cardifolia Linn.) }\end{array}$ & $\begin{array}{l}\text { VataPittahara } \\
\text { (Alleviates Vata and } \\
\text { Pitta) }\end{array}$ & $\begin{array}{l}\text { Balya (strength promoter) } \\
\text { Brumhana (nourishment) } \\
\text { Vrushya (aphrodisiac) }\end{array}$ & $\begin{array}{l}\text { Vatavyadhi (diseases of Vata) } \\
\text { Prameha (diabetes) } \\
\text { Kshaya (cachexia) } \\
\text { RaktaPitta (bleeding disorder) }\end{array}$ \\
\hline 18 & $\begin{array}{l}\text { Shalaparni } \\
\text { (Desmodium } \\
\text { gangeticum) }\end{array}$ & $\begin{array}{l}\text { Tridoshahara } \\
\text { (alleviates Vata, Pitta, } \\
\text { Kapha) }\end{array}$ & $\begin{array}{l}\text { Balya (strength promoter) } \\
\text { Vrushya (aphrodisiac) }\end{array}$ & $\begin{array}{l}\text { Jwara (Fever) } \\
\text { Atisara (diarrhea) } \\
\text { Krimi (worm infestation) } \\
\text { Shota (inflammation) } \\
\text { Chardi (vomiting) } \\
\end{array}$ \\
\hline 19 & $\begin{array}{l}\text { Prushniparni }{ }^{21} \\
\text { (Uraria picta Desv.) }\end{array}$ & $\begin{array}{l}\text { Tridoshahara } \\
\text { (alleviates Vata, Pitta, } \\
\text { Kapha) }\end{array}$ & $\begin{array}{l}\text { Vrushya (aphrodisiac) } \\
\text { Deepana (gastro } \\
\text { stimulant) } \\
\text { Grahi (which holds) }\end{array}$ & $\begin{array}{l}\text { Jwara (fever) } \\
\text { Daha (burning sensation) } \\
\text { Trishna (thirst) } \\
\text { Chardi (Vomiting) } \\
\text { Shwasa (dyspnea) }\end{array}$ \\
\hline 20 & $\begin{array}{l}\text { Haridra }^{22} \\
\text { (Curcuma longa Linn.) }\end{array}$ & $\begin{array}{l}\text { KaphaVatahara } \\
\text { (Alleviates Kapha and } \\
\text { Vata) }\end{array}$ & $\begin{array}{l}\text { Vishaghana (anti } \\
\text { poisonous) } \\
\text { Lekhana (scraping) } \\
\text { Varnya (enhances } \\
\text { complexion) }\end{array}$ & $\begin{array}{l}\text { Kushta (skin disease) } \\
\text { Krimi (worm infestation) } \\
\text { Kandu (pruritis) } \\
\text { Pandu (anemia) } \\
\text { Kamala (jaundice) }\end{array}$ \\
\hline 21 & $\begin{array}{l}\text { Daru haridra } \\
\text { (Berberis aristata Dc.) }\end{array}$ & $\begin{array}{l}\text { Kaphahara } \\
\text { (Alleviates Kapha) }\end{array}$ & $\begin{array}{l}\text { Madakari (intoxicating) } \\
\text { Grahi (binding) } \\
\text { Shukra sthambhaka }\end{array}$ & $\begin{array}{l}\text { Nidra Nasha (insomnia) } \\
\text { Klaibya (impotency) } \\
\text { Jwara (fever) }\end{array}$ \\
\hline
\end{tabular}

(C) 2021 EPRA IJRD | Journal DOI: https://doi.org/10.36713/epra2016 | www.eprajournals.com |45 | 


\section{EPRA International Journal of Research and Development (IJRD)}

Volume: 6 | Issue: 7 | July 2021

- Peer Reviewed Journal

\begin{tabular}{|c|c|c|c|c|}
\hline & & & $\begin{array}{l}\text { (preventing early } \\
\text { ejaculation) }\end{array}$ & $\begin{array}{l}\text { Atisara (diarrhea) } \\
\text { Kasa (cough) }\end{array}$ \\
\hline 22 & $\begin{array}{l}\text { Bruhati }^{24} \\
\text { (Solanum indicum } \\
\text { Linn.) }\end{array}$ & $\begin{array}{l}\text { KaphaVatahara } \\
\text { (Alleviates Kapha and } \\
\text { Vata) }\end{array}$ & $\begin{array}{l}\text { Shukra rechaka (assist } \\
\text { easy ejaculation of } \\
\text { sperm) }\end{array}$ & $\begin{array}{l}\text { Hrudroga (cardiac ailments) } \\
\text { Kushta (skin disease) } \\
\text { Kandu (pruritis) } \\
\text { Krimi (worm infestation) } \\
\text { Shwasa (dyspnea) } \\
\text { Jwara (fever) }\end{array}$ \\
\hline 23 & $\begin{array}{l}\text { Kantakari }^{25} \\
\text { (Solanum } \\
\text { xanthocarpum } \\
\text { Schrad.) }\end{array}$ & $\begin{array}{l}\text { KaphaVatahara } \\
\text { (Alleviates Kapha and } \\
\text { Vata) }\end{array}$ & $\begin{array}{l}\text { Deepana } \\
\text { (gastro-stimulant) } \\
\text { Pachana (digestive) } \\
\text { Ashmarighna } \\
\text { (lithotriptic) } \\
\text { Mutrala (diuretic) } \\
\text { Shukra rechaka (assist } \\
\text { easy ejaculation of } \\
\text { sperm) }\end{array}$ & $\begin{array}{l}\text { Ashmari (calculi) } \\
\text { Mutrakricchra (dysuria) } \\
\text { Shota (inflammation) } \\
\text { Shwasa (dyspnea) } \\
\text { Kasa (cough) }\end{array}$ \\
\hline 24 & $\begin{array}{l}\text { Shwetha Sariva }{ }^{26} \\
\text { (Hemedismus indicus } \\
\text { R. Br.) }\end{array}$ & \multirow[t]{2}{*}{\begin{tabular}{|l|} 
Tridoshahara \\
(alleviates Vata, Pitta, \\
Kapha)
\end{tabular}} & Grahi (binding) & \multirow{2}{*}{$\begin{array}{l}\text { Aruchi (anorexia) } \\
\text { Prameha (diabetes) } \\
\text { Kandu (pruritis) } \\
\text { Jwara (fever) } \\
\text { Atisara (diarrhea) }\end{array}$} \\
\hline 25 & $\begin{array}{l}\text { Krishna Sariva }{ }^{26} \\
\text { (Ichnocarpus } \\
\text { frutescens } R \text {. Br.) }\end{array}$ & & & \\
\hline
\end{tabular}

\section{METHOD OF PREPARATION: ${ }^{27}$}

Equal quantities of each of the drugs are powdered separately, mixed, and made into the form of a paste. This is to be added to 4 parts of Ghrita ( ghee) and 16 parts of water. It has to be cooked on medium heat till the water portion gets evaporated and Ghrita ( ghee) becomes free from froth. Then it is filtered and stored in an airtight container.

\section{INDICATION: $:^{28}$}

Sthavara visha( plant poison), Jangama visha( animal poison), Kritrima visha( artificial poison) and all types of Visha( poison) conditions.

\section{PROBABLE MODE OF ACTION}

Based on Veerya ( potency) of ingredients: $56 \%$ of the ingredients are having Ushna ( hot) Veerya and $44 \%$ of the ingredients are having Sheeta ( cold) Veerya.

Based on the Doshaghnata ( action on dosha) of ingredients:

The majority of the ingredients of this formulation is having Kapha-Vatahara ( alleviates Kapha and Vata) and Tridoshahara ( alleviates Vata, Pitta, Kapha) property.

\section{DISCUSSION}

Ajeya ghrita is indicated in Garavisha ( artificial poison) and all types of Visha ( poison).
This formulation contains twenty-five drugs, out of which 14 drugs have Ushna ( hot) Veerya and 11 drugs have Sheeta ( cold) Veerya. Most of the drugs have Laghu guna ( light) which helps to act fast and Snigdha Guna ( unctuous) of the ingredients helps to counteract the poison. Most of the drugs have KaphaVatahara ( alleviates Kapha and Vata) and Tridoshahara ( alleviates Kapha and Vata) properties which help in counteracting the ill effects caused because of Visha ( poison). The drugs in Ajeya ghrita have Vishaghna ( anti-poisonous) property and the symptoms of Garavisha like Krisha ( cachexia), Alpagni ( weak digestive power), Shwasa ( dyspnea), Kasa ( cough), Jwara ( fever), Shotha ( inflammation) are counteracted by Brumhana ( nourishment), Deepana ( gastro-stimulant), Shwasa Kasahara ( alleviates dyspnea and cough), Jwarahara ( anti pyretic), Shothahara ( anti-inflammatory) properties of the particular ingredients. Ajeya ghrita is been proved for its cardioprotective (Hrudayavarana) activity due to its ability to increase anti-oxidant property in the form of catalase activity and to reduce lipid peroxidation of the myocardial cell membrane. It prevents cell damage due to its anti-oxidant property responsible for a free radical generation. ${ }^{29}$ Ghrita ( ghee) by nature it is Tridoshahara ( alleviates Kapha and Vata) and has Vishaghna ( anti-poisonous) property. ${ }^{30}$ Ghrita ( ghee) is used as an ingredient or as Anupana in most anti-toxic preparations. Ghrita 


\section{SJIF Impact Factor 2021: 8.013| ISI I.F.Value:1.241| Journal DOI: 10.36713/epra2016 ISSN: 2455-7838(Online) EPRA International Journal of Research and Development (IJRD)}

Volume: 6 | Issue: 7 | July 2021

( qghee) contains Vit A and Vit $\mathrm{E}$ which help to reduce the ketone bodies. Ghrita ( ghee) contains Betacarotene which has anti-oxidant properties. ${ }^{31}$

\section{CONCLUSION}

When Visha ( poison) enters the body it first affects Hridaya ( heart) and affects the life of the person. Thus administration of Ghrita ( ghee) eliminates the effect of Visha ( poison) and protects the heart. Hence Ghrita Kalpa ( ghee preparation) has a significant role in the treatment of Visha ( poison). Also, the ingredients of Ajeya Ghrita have Vishahara ( anti-poisonous) properties, which makes the formulation more effective.

\section{REFERENCE}

1. Ambikadatta S, Sushrutha Samhita of Sushrutha, Sutra sthana; Vedotpatti adhyaya: Chapter 1, Verse 14. Varanasi: Chaukambha samskruta samsthana, Reprint 2014;p.6

2. Paradkar BH, editor, $6^{\text {th }}$ edition. Ashtanga hridaya of Vagbhata, Uttara sthana; Vishapratisheda: Chapter 35, Verse 49. Bombay: Pandurang jawaji,1939;p.905

3. Shastry JLN. Illustrated dravyaguna Vijnana. $1^{\text {st }}$ ed. Golhar, Varanasi: Chaukambha orientalia;2003, Reprint 2010, Vol 2:p.824-825

4. Shastry JLN. Illustrated dravyaguna Vijnana. $1^{\text {st }}$ ed. Golhar, Varanasi: Chaukambha orientalia;2003, Reprint 2010, Vol 2:p.801-802

5. Shastry JLN. Illustrated dravyaguna Vijnana. $1^{\text {st }}$ ed. Golhar, Varanasi: Chaukambha orientalia;2003, Reprint 2010, Vol 2:p.307-313

6. Shastry JLN. Illustrated dravyaguna Vijnana. $1^{\text {st }}$ ed. Golhar, Varanasi: Chaukambha orientalia;2003, Reprint 2010, Vol 2:p.507-509

7. Sharma PV, editor $2009^{\text {th }}$ edition. Kaiyadeva nighantu; dhanya Varga. Verse 67-69. Varanasi: Chaukambha orientalia

8. Shastry JLN. Illustrated dravyaguna Vijnana. $1^{\text {st }}$ ed. Golhar, Varanasi: Chaukambha orientalia;2003, Reprint 2010, Vol 2:p.277-281

9. Shastry JLN. Illustrated dravyaguna Vijnana. $1^{\text {st }}$ ed. Golhar, Varanasi: Chaukambha orientalia;2003, Reprint 2010, Vol 2:p.527-530

10. Mishra B, Vaishya $R$, editors. Bhavaprakasha Samhita(Vidyotini hindi commentary) of Bhavamishra. $10^{\text {th }}$ ed. Varanasi: Chaukambha Sanskrit Sansthan; 2002. Karpuradi Varga

11. Shastry JLN. Illustrated dravyaguna Vijnana. $1^{\text {st }}$ ed. Golhar, Varanasi: Chaukambha orientalia;2003, Reprint 2010, Vol 2:p.67-77

12. Shastry JLN. Illustrated dravyaguna Vijnana. $1^{\text {st }}$ ed. Golhar, Varanasi: Chaukambha orientalia;2003, Reprint 2010, Vol 2:p.587-588
- Peer Reviewed Journal

13. Shastry JLN. Illustrated dravyaguna Vijnana. $1^{\text {st }}$ ed. Golhar, Varanasi: Chaukambha orientalia;2003, Reprint 2010, Vol 2:p.551-557

14. Shastry JLN. Illustrated dravyaguna Vijnana. $1^{\text {st }}$ ed. Golhar, Varanasi: Chaukambha orientalia;2003, Reprint 2010, Vol 2:p.318-320

15. Shastry JLN. Illustrated dravyaguna Vijnana. $1^{\text {st }}$ ed. Golhar, Varanasi: Chaukambha orientalia;2003, Reprint 2010, Vol 2:p.478-482

16. Shastry JLN. Illustrated dravyaguna Vijnana. $1^{\text {st }}$ ed. Golhar, Varanasi: Chaukambha orientalia;2003, Reprint 2010, Vol 2:p.510-512

17. Shastry JLN. Illustrated dravyaguna Vijnana. $1^{\text {st }}$ ed. Golhar, Varanasi: Chaukambha orientalia;2003, Reprint 2010, Vol 2:p.893-894

18. Shastry JLN. Illustrated dravyaguna Vijnana. $1^{\text {st }}$ ed. Golhar, Varanasi: Chaukambha orientalia;2003, Reprint 2010, Vol 2:p.1038-1039

19. Shastry JLN. Illustrated dravyaguna Vijnana. $1^{\text {st }}$ ed. Golhar, Varanasi: Chaukambha orientalia;2003, Reprint 2010, Vol 2:p.87-90

20. Shastry JLN. Illustrated dravyaguna Vijnana. $1^{\text {st }}$ ed. Golhar, Varanasi: Chaukambha orientalia;2003, Reprint 2010, Vol 2:p.160-163

21. Shastry JLN. Illustrated dravyaguna Vijnana. $1^{\text {st }}$ ed. Golhar, Varanasi: Chaukambha orientalia;2003, Reprint 2010, Vol 2:p.164-166

22. Shastry JLN. Illustrated dravyaguna Vijnana. $1^{\text {st }}$ ed. Golhar, Varanasi: Chaukambha orientalia;2003, Reprint 2010, Vol 2:p.513-518

23. Shastry JLN. Illustrated dravyaguna Vijnana. $1^{\text {st }}$ ed. Golhar, Varanasi: Chaukambha orientalia;2003, Reprint 2010, Vol 2:p.54-56

24. Shastry JLN. Illustrated dravyaguna Vijnana. $1^{\text {st }}$ ed. Golhar, Varanasi: Chaukambha orientalia;2003, Reprint 2010, Vol 2:p.371-374

25. Shastry JLN. Illustrated dravyaguna Vijnana. $1^{\text {st }}$ ed. Golhar, Varanasi: Chaukambha orientalia;2003, Reprint 2010, Vol 2:p.367-370

26. Shastry JLN. Illustrated dravyaguna Vijnana. $1^{\text {st }}$ ed. Golhar, Varanasi: Chaukambha orientalia;2003, Reprint 2010, Vol 2:p.348-351

27. Tripathi B, Sharangadhar S, Dipika Hindi Vyakhya. Varanasi: Chaukambha Surbharati Prakashan; 2004. Madhyama Khanda 9/1, 9/3-4, 9/8-11, 9/12$15,9 / 18$.

28. Mishra J, Sharma S, Ashtanga Sangraha of Vrudha Vagbhata, Uttara tantra; Vishapratisheda: Chapter 40, Verse 73. Varanasi: Chaukambha Sanskrit series office; $p .854$

29. Cholakkal, H.(2018)/118. An experimental study to evaluate the cardioprotective activity of Ajeya ghrita in Albino rats. Journal of Ayurveda and Integrated Medical Sciences (JAIMS), 9(2), S19. https://doi.org/10.1016/j.jaim.2018.02.081

30. Garde GK. Sartha Vagbhata, Sutrasthana; Dravadravyavignaniyam: Chapter 5, Verse 37-39. Varanasi: Chaukambha Subharati Publication;p.20 


\section{EPRA International Journal of Research and Development (IJRD)}

Volume: 6 | Issue: 7 | July 2021

\section{- Peer Reviewed Journal}

31. BN, D.A.K, Dixit,S.O., \& Patel,S.B. (2016). A

Critical Review on Go-Ghrita, Shata Dhouta Ghrita and Sahasra Dhouta Ghrita. Journal of Ayurveda and Integrated Medical Sciences (JAIMS), 1(3).

32. https://doi.org/10.21760/jaims.v1i3.4427

33. Paradkar, B.H., editor, $6^{\text {th }}$ edition. Ashtanga hridaya of Vagbhata, Uttara sthana; Vishapratisheda: Chapter 35, Verse 9-10. Bombay:

Pandurang jawaji,1939;p.903 